\title{
Neuro-Fuzzy Speed Tracking Control of Traveling-Wave Ultrasonic Motor Drives Using Direct Pulsewidth Modulation
}

\author{
K. T. Chau, Member, IEEE, S. W. Chung, and C. C. Chan, Fellow, IEEE
}

\begin{abstract}
The traveling-wave ultrasonic motor (TWUM) drive offers many distinct advantages but suffers from severe system nonlinearities and parameter variations, especially during speed control. This paper presents a new speed tracking control system for the TWUM drive, which newly incorporates neuro-fuzzy control and direct pulsewidth modulation to solve the problem of nonlinearities and variations. The proposed control system is digitally implemented by a low-cost digital-signal-processor-based microcontroller, hence reducing the system hardware size and cost. Experimental results confirm that the proposed speed tracking controller can offer good steady-state and transient performance.
\end{abstract}

Index Terms-Neuro-fuzzy control, pulsewidth modulation (PWM), ultrasonic motor.

\section{INTRODUCTION}

$\mathbf{R}$ ECENTLY, the traveling-wave ultrasonic motor (TWUM) drive has become attractive for mechatronic applications because of its advantages of high torque at low speeds, light weight, compact size, fast response, no electromagnetic interference (EMI), quiet operation, and self-locking capability [1]. Also, it is very suitable for gearless drive applications. However, the TWUM drive exhibits heavily nonlinear behavior, and high sensitivity to temperature change. The drift of temperature-dependent parameters is particularly significant during the speed tracking control for prolonged operation.

The motion control of the TWUM can be accomplished by controlling the driving frequency, the phase difference, and the voltage amplitude of two sinusoidal voltage waveforms applied to the motor terminals. These three control strategies have their own advantages and disadvantages. Firstly, the driving frequency control strategy (typically from 40 to $43 \mathrm{kHz}$ ) takes the advantage of simple analog hardware implementation by using a voltage controlled oscillator, but suffers from a sensitivity problem which is caused by the very narrow range of operating frequencies. Secondly, the phase difference control strategy (typically from $-90^{\circ}$ to $90^{\circ}$ ) can offer the merit of bidirectional rotation, but may create severe audible noise at

Paper IPCSD 03-034, presented at the 2002 Industry Applications Society Annual Meeting, Pittsburgh, PA, October 13-18, and approved for publication in the IEEE TRANSACTIONS ON INDUSTRY APPLICATIONS by the Industrial Drives Committee of the IEEE Industry Applications Society. Manuscript submitted for review October 15, 2002 and released for publication April 9, 2003. This work was supported by the Hong Kong Research Grants Council under Project HKU 7039/00E.

The authors are with The University of Hong Kong, Hong Kong (e-mail: ktchau@eee.hku.hk; swchung@eee.hku.hk; ccchan@eee.hku.hk).

Digital Object Identifier 10.1109/TIA.2003.813736 the instant of phase difference changes. Thirdly, the voltage amplitude control strategy (typically, from 100 to $130 \mathrm{~V}$ ) can offer the most linear speed control characteristics among the three control methods, but it suffers from a limited speed range at a particular frequency. Also, it usually involves an additional boost converter to adjust the dc-link voltage of the two-phase inverter.

Both the driving frequency control strategy and the phase difference control strategy were widely applied to motion control of the TWUM [2]-[4], whereas the voltage amplitude control strategy using a boost converter was seldom employed [5]. Abandoning the use of a boost converter, the voltage amplitude control strategy could be conducted by pulsewidth modulation (PWM). In [6], the voltage amplitude was adjusted by the forward-and-backward PWM method for position control of the TWUM. In [7], the voltage amplitude was adjusted by the ON-OFF PWM method for speed control. Both methods essentially adopted the same principle in which the modulation was to control the ratio of the number of ultrasonic cycles within the forward-direction or on-time duration to the total number of ultrasonic cycles including the backward-direction or off-time duration. Such control methods cause intermittent operation of the TWUM, leading to the creation of severe audible noise. In order to suppress this audible noise, the PWM should be applied to each ultrasonic cycle [8].

Apart from adopting different control strategies to achieve a wide speed range for the TWUM, different control algorithms need to be considered to solve the problems of heavy nonlinearity and high temperature sensitivity. Although many attempts have been made to model the TWUM, the resulting models are so complex or data demanding that they are impractical for real-time control [9]-[11]. Alternatively, many empirical approaches, such as proportional integral (PI) control [12], fuzzy logic control [13], adaptive control [14], and neural network control [15], have been proposed for motion control of the TWUM. The PI control is simple and offers a wide stability margin. However, it requires meticulous fine tuning and cannot cope with system parameter variations. The fuzzy logic control can compensate the system nonlinearities through human expertise. Yet, it relies too much on the intuition and experience of the designer. The adaptive control can self-adjust the controller parameters to adapt system parameter variations. Unfortunately, it generally requires a reference model of the system, which is impractical for the TWUM. The neural network control can handle the complicated nonlinear characteristics of the system, but suffers from the problem of lengthy 
training and convergence time. There is a trend of employing neuro-fuzzy control (NFC), also called fuzzy neural network control (FNNC) for motion control of the TWUM [4], [16], [17]. This NFC exhibits the fuzzy logic to vaguely describe the should-be behavior, and the neural network to compensate any parameter variations. However, there are difficulties in establishing the fuzzy rules to describe the should-be behavior [4].

In this paper, a new dual-mode NFC controller is proposed and implemented for speed tracking control of the TWUM. The key is to newly incorporate NFC and direct PWM as well as to simultaneously employ both the driving frequency and voltage amplitude as the dual-mode control variables to handle system nonlinearities and parameter variations. In order to reduce the system hardware size and cost, the proposed controller is implemented by a low-cost single-chip digital-signal-processor (DSP)-based microcontroller.

Compared with the previous work [4] in which a complicated dual-mode NFC controller was developed, this paper offers the definite advantage that the proposed dual-mode NFC controller can provide piecewise-linear control over the whole speed range, hence solving the severe nonlinearity and audible noise problem associated with the phase difference control. Also, the proposed dual-mode control variables can reduce the complexity of NFC structure, hence simplifying the formulation of fuzzy rules. Compared with the previous work [8] in which a dual-mode PI controller was developed, the proposed dual-mode NFC controller can adapt the system parameter variations which cannot be handled by a dual-mode PI controller.

In Section II, the operating principle and characteristics of the TWUM will be briefly described. Then, the NFC and direct PWM will be described in Sections III and IV, respectively. Section $\mathrm{V}$ will be devoted to discussing the setup of the proposed speed tracking control system. In Section VI, experimental results will be given to verify the effectiveness of the proposed controller. Finally, conclusions will be drawn in Section VII.

\section{OPERATING PRINCIPLE AND ChARACTERISTICS}

Instead of using the principle of electromagnetism, the TWUM utilizes the piezoelectric effect to convert electrical energy into mechanical motion [1]. Thus, its operating principle is fundamentally different from that of electromagnetic motors. As shown in Fig. 1, piezoelectric elements are positioned at an appropriate distance from one another. One is fed by the phase- $A$ voltage source $V_{m} \sin \omega t$ to produce a mechanical vibration while another is fed by the phase- $B$ voltage source $V_{m} \sin (\omega t+\phi)$ to generate another vibration, where $V_{m}$ is the amplitude, $\omega=2 \pi f$ is the angular version of driving frequency $f$, and $\phi$ is the phase difference of the two voltage sources. Provided that the phase difference is positive, a traveling wave propagating to the right-hand direction is generated in the stator. Making use of a spring to keep a rigid rotor in pressure contact with the stator, the rotor can be driven to travel in the left-hand direction by the tangential force at the contact surface resulting from the elliptical movements at the wave crests. Although the rotor moves only about a micrometer in

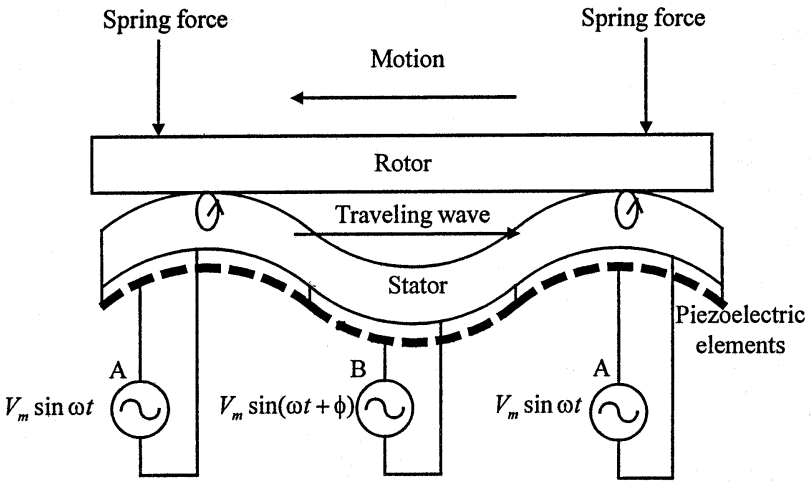

Fig. 1. Operating principle.

an individual cycle of the elliptical motion, this can add up to a speed $N$ of several centimeters per second at ultrasonic frequencies. By connecting the ends of the TWUM to form a ring, the corresponding rotational speed can achieve up to several hundred revolutions per minute. Without excitation by the proper electrical power source, the rotor cannot rotate as it is pressed against the stator, hence producing a self-locking capability.

Typical nonlinear speed characteristics of the TWUM are illustrated in Fig. 2. It can be found that the relationship between $N$ and $f$ is highly nonlinear, and the controllable range of $f$ is seriously dependent on the load torque; the relationship between $N$ and $\phi$ not only depends on $f$, but also exhibits frequency-varying dead zones at near-zero $\phi$; the relationship between $N$ versus $V_{m}$ (as controlled by duty ratio $d$ ) is essentially linear at a given $f$. Therefore, it is anticipated that the dual-mode control (simultaneously controlling $f$ and $d$ ) can offer piecewise-linear characteristics over a wide speed range.

\section{NFC}

Recently, a number of NFC schemes have been proposed, focusing on the variations of structure and parameter learning. Fig. 3 shows the NFC structure [18] for the proposed speed tracking control of the TWUM. As depicted in Fig. 3(a), the command signal $y_{c}$ is first compared with the feedback signal $y$, then the corresponding error $\varepsilon$ is input into the NFC controller, hence computing the desired driving frequency change $\Delta f$ and duty ratio change $\Delta d$. As shown in Fig. 3(b), the NFC controller is based on a five-layered structure. The node in the layer 1 (called the input layer) simply directs the crisp input state $($ namely, $\varepsilon$ ) to layer 2 . The nodes in layer 2 are input term nodes representing the input linguistic variables. They act as characteristic functions to transform the crisp value into fuzzy numbers. Hence, fuzzification is performed in this layer. Each node in layer 3 is a rule node that represents one fuzzy logic rule. As a result, all the layer-3 nodes form a fuzzy rule base. The nodes in layer 4 are output term nodes representing the output linguistic variables. They serve to integrate the rules which have the same consequence. Finally, the nodes in layer 5 (called the output layer) convert the resulting fuzzy numbers into the crisp output control signals ( $\Delta f$ and $\Delta d$ ). Hence, the defuzzification is done in this layer. 


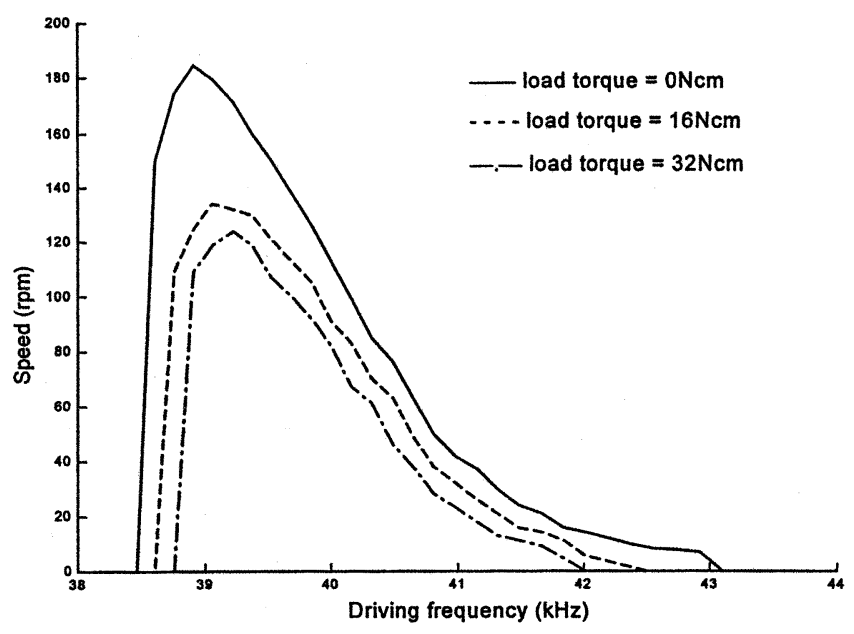

(a)

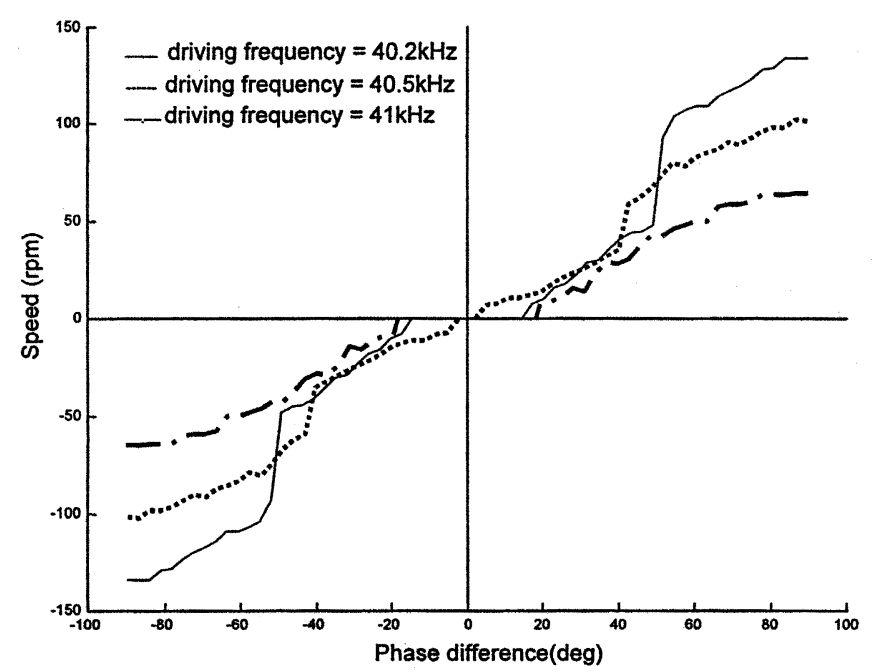

(b)

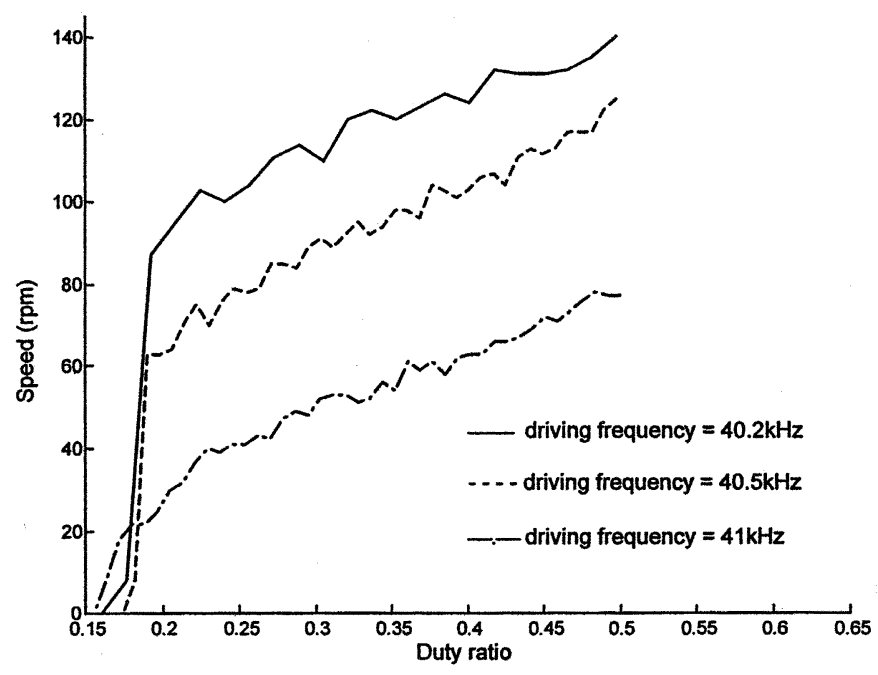

(c)

Fig. 2. Speed characteristics. (a) Speed versus driving frequency. (b) Speed versus phase difference. (c) Speed versus duty ratio.

Based on this NFC structure, the net input to the node $i$ in layer $k$ can be represented by an integration function $F_{i}^{(k)}\left(u_{1}^{(k)}, u_{2}^{(k)}, \ldots, u_{p}^{(k)} ; w_{1}^{(k)}, w_{2}^{(k)}, \ldots, w_{p}^{(k)}\right)$ to combine the information from other nodes, where $u_{1}^{(k)}, u_{2}^{(k)}, \ldots, u_{p}^{(k)}$ are inputs to this node and $w_{1}^{(k)}, w_{2}^{(k)}, \ldots, w_{p}^{(k)}$ are the associated link weights. Then, the output of this node is given by $o_{i}^{(k)}=a_{i}^{(k)}\left(F_{i}^{(k)}\right)$ where $a_{i}^{(k)}$ is the activation function of the node $i$ in the layer $k$ [18].

As each node in layer 1 simply directs the crisp input value to the layer 2, the output $o_{i}^{(1)}$ is identical to its input $u_{i}^{(1)}$, yielding

$$
\begin{aligned}
& F_{i}^{(1)}=u_{i}^{(1)} \\
& a_{i}^{(1)}=F_{i}^{(1)} .
\end{aligned}
$$

Hence, the layer- 1 link weight $w_{i}^{(1)}$ is always unity. In the proposed application, $u_{1}^{(1)}$ is $\varepsilon$.

In layer 2, the fuzzification process transforms a crisp input $u_{i}^{(2)}$ into a fuzzy output $o_{i}^{(2)}$ which is characterized by its de- gree of belonging to the $j$ th term of the $i$ th input linguistic variable $x_{i}$, based on the user-specified membership function $M_{x_{i}}^{j}$. When a bell-shaped characteristic function is adopted, the fuzzified output in this layer is given by

$$
o_{i}^{(2)}=M_{x_{i}}^{j}\left(u_{i}^{(2)}, m_{i j}^{(2)}, \sigma_{i j}^{(2)}\right)=\exp \left(-\frac{\left(u_{i}^{(2)}-m_{i j}^{(2)}\right)^{2}}{\left(\sigma_{i j}^{(2)}\right)^{2}}\right)
$$

where $m_{i j}^{(2)}$ and $\sigma_{i j}^{(2)}$ are the mean and the variance of the characteristic function, respectively. Hence, the corresponding integration and activation functions can be expressed as

$$
\begin{aligned}
& F_{i}^{(2)}=-\frac{\left(u_{i}^{(2)}-m_{i j}^{(2)}\right)^{2}}{\left(\sigma_{i j}^{(2)}\right)^{2}} \\
& a_{i}^{(2)}=\exp \left(F_{i}^{(2)}\right) .
\end{aligned}
$$




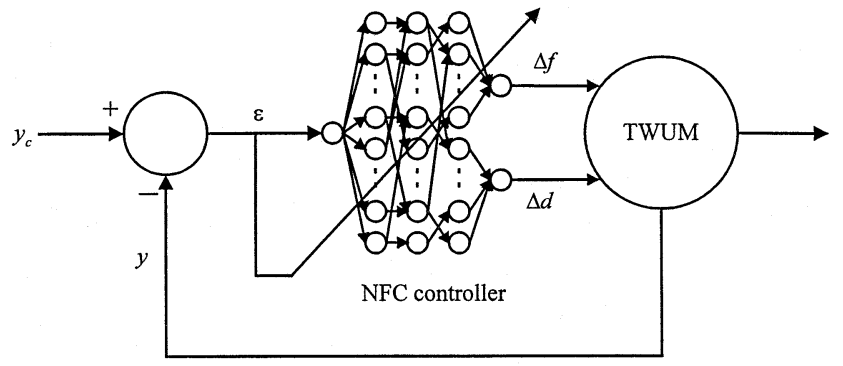

(a)

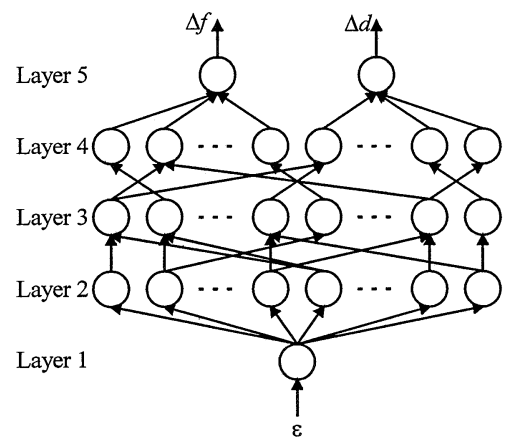

(b)

Fig. 3. NFC approach. (a) System configuration. (b) Controller structure.

TABLE I

FUZZY CONTROL RULES

\begin{tabular}{cccccccc}
\hline$\varepsilon$ & NB & NM & NS & ZO & PS & PM & PB \\
$\Delta f$ & PB & PM & PS & ZO & NS & NM & NB \\
$\Delta d$ & NB & NM & NS & ZO & PS & PM & PB \\
\hline
\end{tabular}

Thus, the layer-2 link weight $w_{i j}^{(2)}$ is governed by both $m_{i j}^{(2)}$ and $\sigma_{i j}^{(2)}$. In this application, the input linguistic variables of $\varepsilon$ are described by the bell-shaped characteristic functions.

As the links in layer 3 are used to perform precondition matching of fuzzy logic rules, those rule nodes carry out the fuzzy AND operation. In this application, the rules are tabulated in Table I, which are established according to the speed characteristics in Fig. 2 and the should-be behavior of a motor controller. As a result, the fuzzy input $u_{i}^{(3)}$ can be directly mapped to the fuzzy output $o_{i}^{(3)}$. Hence, it yields

$$
\begin{aligned}
& F_{i}^{(3)}=u_{i}^{(3)} \\
& a_{i}^{(3)}=F_{i}^{(3)} .
\end{aligned}
$$

The layer-3 link weight $w_{i}^{(3)}$ is then always unity. For each rule node, there is at most one link (maybe none) from those term nodes of a linguistic node. By describing rules in a human way, the nonlinear relationship between $(\Delta f, \Delta d)$ and $\varepsilon$ can be easily embedded into the controller structure.

It should be noted that the fuzzy rules of this NFC controller can be easily formulated because of the straightforward should-be behavior of the proposed single-input NFC structure, namely, the relationship between $(\Delta f, \Delta d)$ and $\varepsilon$. It is a definite advantage over the previous dual-input NFC structure [4] in which the should-be behavior between $(\Delta f, \Delta \phi)$ and $(\varepsilon$, $\omega$ ) was complex and hence the formulation of fuzzy rules was much more difficult.
In layer 4, the links perform the fuzzy OR operation to integrate the rules which have the same consequence. The fuzzy output $o_{i}^{(4)}$ can thus be evaluated as the summation of those fuzzy inputs $u_{1}^{(4)}, u_{2}^{(4)}, \ldots, u_{p}^{(4)}$, but bounded by unity. Hence, the corresponding integration and activation functions are given by

$$
\begin{aligned}
F_{i}^{(4)} & =\sum_{i} u_{i}^{(4)} \\
a_{i}^{(4)} & =\min \left(1, F_{i}^{(4)}\right) .
\end{aligned}
$$

Also, the layer-4 link weight $w_{i}^{(4)}$ is always unity.

Finally, the linguistic control signals $\Delta f$ and $\Delta d$ in layer 5 need to be converted back to the crisp values. By adopting the center-of-area defuzzification method [18], the defuzzified output is given by

$$
o_{i}^{(5)}=\frac{\sum_{j}\left(m_{i j}^{(5)} \sigma_{i j}^{(5)}\right) u_{i j}^{(5)}}{\sum_{j} \sigma_{i j}^{(5)} u_{i j}^{(5)}}
$$

where $u_{i j}^{(5)}$ is the input of the $j$ th term of the $i$ th output linguistic variable, while $m_{i j}^{(5)}$ and $\sigma_{i j}^{(5)}$ are the corresponding mean and variance of the bell-shaped membership function, respectively. Hence, it yields

$$
\begin{aligned}
F_{i}^{(5)} & =\sum_{j}\left(m_{i j}^{(5)} \sigma_{i j}^{(5)}\right) u_{i j}^{(5)} \\
a_{i}^{(5)} & =\frac{F_{i}^{(5)}}{\sum_{j} \sigma_{i j}^{(5)} u_{i j}^{(5)}} .
\end{aligned}
$$

Thus, the layer-5 link weight $w_{i j}^{(5)}$ is $m_{i j}^{(5)} \sigma_{i j}^{(5)}$. In this application, the bell-shaped characteristic functions are used to describe the output linguistic variables $\Delta f$ and $\Delta d$.

Therefore, this NFC structure possesses the features of fuzzy logic control due to the operation of linguistic variables, characteristic functions and fuzzy control rules, as well as the symptoms of neural network control due to the structure involving nodes, layers, and weights.

Having established the NFC structure, the network enters the learning phase to adjust the parameters of the input and output membership functions optimally. The backpropagation algorithm is adopted for this supervised learning [18]. The goal is to minimize the error function $E$ as given by

$$
E=\frac{1}{2}\left(y_{c}(t)-y(t)\right)^{2}=\frac{1}{2} \varepsilon^{2} .
$$

Then, starting at the output nodes, a backward pass is used to compute $\partial E / \partial w$ for all the hidden nodes. The corresponding general learning rule is expressed as

$$
w(t+T)=w(t)+\eta\left(-\frac{\partial E}{\partial w}\right)
$$

where $\eta$ is the learning rate and $T$ is the interval for successive update. 
In layer $5, w_{i j}^{(5)}$ is $m_{i j}^{(5)} \sigma_{i j}^{(5)}$. The adaptive rules of $m_{i j}^{(5)}$ and $\sigma_{i j}^{(5)}$ are derived as

$$
\begin{aligned}
\frac{\partial E}{\partial m_{i j}^{(5)}} & =\frac{\partial E}{\partial a_{i}^{(5)}} \frac{\partial a_{i}^{(5)}}{\partial m_{i j}^{(5)}} \\
\frac{\partial E}{\partial \sigma_{i j}^{(5)}} & =\frac{\partial E}{\partial a_{i}^{(5)}} \frac{\partial a_{i}^{(5)}}{\partial \sigma_{i j}^{(5)}}
\end{aligned}
$$

Substituting $y(t)=a_{i}^{(5)}$ into (13), $\partial E / \partial a_{i}^{(5)}$ can be obtained as

$$
\frac{\partial E}{\partial a_{i}^{(5)}}=-\left(y_{c}(t)-y(t)\right)=-\varepsilon .
$$

By using (11) and (12), $\partial a_{i}^{(5)} / \partial m_{i j}^{(5)}$ can be obtained as

$$
\frac{\partial a_{i}^{(5)}}{\partial m_{i j}^{(5)}}=\frac{\sigma_{i j}^{(5)} u_{i j}^{(5)}}{\sum_{j} \sigma_{i j}^{(5)} u_{i j}^{(5)}}
$$

Hence, by substituting (17) and (18) into (15), and then using (14), $m_{i j}^{(5)}$ is updated by

$$
m_{i j}^{(5)}(t+T)=m_{i j}^{(5)}(t)+\eta \varepsilon \frac{\sigma_{i j}^{(5)} u_{i j}^{(5)}}{\sum_{j} \sigma_{i j}^{(5)} u_{i j}^{(5)}} .
$$

Similarly, by using (11), (12), (14), (16), and (17), $\sigma_{i j}^{(5)}$ is updated by

$$
\begin{aligned}
& \sigma_{i j}^{(5)}(t+T)=\sigma_{i j}^{(5)}(t) \\
& +\eta \varepsilon \frac{m_{i j}^{(5)} u_{i j}^{(5)} \sum_{j} \sigma_{i j}^{(5)} u_{i j}^{(5)}-u_{i j}^{(5)} \sum_{j} m_{i j}^{(5)} \sigma_{i j}^{(5)} u_{i j}^{(5)}}{\left(\sum_{j} \sigma_{i j}^{(5)} u_{i j}^{(5)}\right)^{2}} .
\end{aligned}
$$

By using (17), the error $\delta_{i}^{(5)}$ to be propagated to layer 4 is given by

$$
\delta_{i}^{(5)}=-\frac{\partial E}{\partial a_{i}^{(5)}}=\varepsilon
$$

Since $w_{i}^{(4)}$ in layer 4 is always unity, no weight adjustment is needed. Nevertheless, the error $\delta_{i}^{(4)}$ needs to be computed and propagated as given by

$$
\delta_{i}^{(4)}=-\frac{\partial E}{\partial a_{i}^{(4)}}
$$

Since $a_{i}^{(4)}=u_{i j}^{(5)},(22)$ can be rewritten as

$$
\delta_{i}^{(4)}=-\frac{\partial E}{\partial a_{i}^{(5)}} \frac{\partial a_{i}^{(5)}}{\partial u_{i j}^{(5)}}
$$

By using (11) and (12), $\partial a_{i}^{(5)} / \partial u_{i j}^{(5)}$ can be obtained. Hence, making use of (21), (23) can be expressed as

$$
\delta_{i}^{(4)}=\varepsilon \frac{m_{i j}^{(5)} \sigma_{i j}^{(5)} \sum_{j} \sigma_{i j}^{(5)} u_{i j}^{(5)}-\sigma_{i j}^{(5)} \sum_{j} m_{i j}^{(5)} \sigma_{i j}^{(5)} u_{i j}^{(5)}}{\left(\sum_{j} \sigma_{i j}^{(5)} u_{i j}^{(5)}\right)^{2}} .
$$

Similarly to layer 4 , there is no weight to be adjusted in layer 3 . By employing $a_{i}^{(3)}=u_{i}^{(4)}, a_{i}^{(4)}=u_{i}^{(4)}$ and (22), the error $\delta_{i}^{(3)}$ can be easily obtained as

$$
\delta_{i}^{(3)}=-\frac{\partial E}{\partial a_{i}^{(3)}}=-\frac{\partial E}{\partial a_{i}^{(4)}} \frac{\partial a_{i}^{(4)}}{\partial u_{i}^{(4)}}=\delta_{i}^{(4)} .
$$

In layer 2 , similarly to layer 5 , the adaptive rules of $m_{i j}^{(2)}$ and $\sigma_{i j}^{(2)}$ are derived as

$$
\begin{aligned}
\frac{\partial E}{\partial m_{i j}^{(2)}} & =\frac{\partial E}{\partial a_{i}^{(2)}} \frac{\partial a_{i}^{(2)}}{\partial m_{i j}^{(2)}} \\
\frac{\partial E}{\partial \sigma_{i j}^{(2)}} & =\frac{\partial E}{\partial a_{i}^{(2)}} \frac{\partial a_{i}^{(2)}}{\partial \sigma_{i j}^{(2)}}
\end{aligned}
$$

Since $a_{i}^{(2)}=u_{i}^{(3)}$ and using (25), the error $\delta_{i}^{(2)}$ is given by

$$
\delta_{i}^{(2)}=-\frac{\partial E}{\partial a_{i}^{(2)}}=-\frac{\partial E}{\partial u_{i}^{(3)}}=-\frac{\partial E}{\partial a_{i}^{(3)}} \frac{\partial a_{i}^{(3)}}{\partial u_{i}^{(3)}}=\delta_{i}^{(3)} \frac{\partial a_{i}^{(3)}}{\partial u_{i}^{(3)}}
$$

From (6) and (7), $\partial a_{i}^{(3)} / \partial u_{i}^{(3)}$ can be obtained. Hence, (28) can be rewritten as

$$
\delta_{i}^{(2)}=\delta_{i}^{(3)}
$$

By using (4) and (5), $\partial a_{i}^{(2)} / \partial m_{i j}^{(2)}$ can be obtained as

$$
\frac{\partial a_{i}^{(2)}}{\partial m_{i j}^{(2)}}=\exp \left(-\frac{\left(u_{i}^{(2)}-m_{i j}^{(2)}\right)^{2}}{\left(\sigma_{i j}^{(2)}\right)^{2}}\right) \frac{2\left(u_{i}^{(2)}-m_{i j}^{(2)}\right)}{\left(\sigma_{i j}^{(2)}\right)^{2}}
$$

Hence, by substituting (28) and (30) into (26), and then using (14), $m_{i j}^{(2)}$ is updated by

$$
\begin{aligned}
& m_{i j}^{(2)}(t+T)=m_{i j}^{(2)}(t) \\
&+\eta \delta_{i}^{(2)} \exp \left(-\frac{\left(u_{i}^{(2)}-m_{i j}^{(2)}\right)^{2}}{\left(\sigma_{i j}^{(2)}\right)^{2}}\right) \frac{2\left(u_{i}^{(2)}-m_{i j}^{(2)}\right)}{\left(\sigma_{i j}^{(2)}\right)^{2}} .
\end{aligned}
$$

Similarly, by using (4), (5), (14), (27), and (28), the update rule of $\sigma_{i j}^{(2)}$ becomes

$$
\begin{aligned}
& \sigma_{i j}^{(2)}(t+T)=\sigma_{i j}^{(2)}(t) \\
&+\eta \delta_{i}^{(2)} \exp \left(-\frac{\left(u_{i}^{(2)}-m_{i j}^{(2)}\right)^{2}}{\left(\sigma_{i j}^{(2)}\right)^{2}}\right) \frac{2\left(u_{i}^{(2)}-m_{i j}^{(2)}\right)^{2}}{\left(\sigma_{i j}^{(2)}\right)^{3}} .
\end{aligned}
$$

Therefore, based on (19), (20), (31), and (32), those weights described by $m_{i j}^{(2)}, \sigma_{i j}^{(2)}, m_{i j}^{(5)}$ and $\sigma_{i j}^{(5)}$ are continually updated by backpropagation. It should be noted that the corresponding 


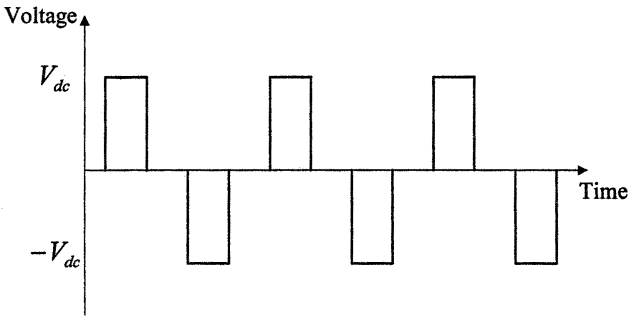

Fig. 4. Direct PWM waveform at $25 \%$ duty ratio.

convergence speed is superior to that of the normal backpropagation scheme for neural networks because expertise has been implanted as characteristic functions and fuzzy rules before the learning phase.

On the other hand, this NFC structure suffers from a problem that it is difficult to relate the motor output error to the controller output error, due to the absence of the mathematical model and, hence, the Jacobian of the TWUM. Although an additional neural network can be incorporated to estimate the Jacobian, it requires heavy computational effort and suffers from difficulty in implementation. In order to solve the problem because of the unknown Jacobian, an approximation of $\partial E / \partial w$ can be employed [19] as given by

$$
\frac{\partial E}{\partial w}=\frac{\partial E}{\partial y} \frac{\partial y}{\partial p} \frac{\partial p}{\partial w} \approx-\varepsilon \operatorname{sgn}\left(\frac{\partial y}{\partial p}\right) \frac{\partial p}{\partial w}
$$

where $p$ is the controller output or TWUM input (namely, $\Delta f$ or $\Delta d$ ). Notice that $\partial p / \partial w$ can be readily obtained through backpropagation, while $\operatorname{sgn}(\partial y / \partial p)$ is generally known during operation.

\section{DIRECT PWM}

Another core of this speed control system lies on the proposed direct PWM method which functions to adjust the pulsewidth of each ultrasonic switching cycle, hence, to regulate the voltage amplitude. This direct PWM method takes definite advantages over the available PWM methods in terms of controllability and audible noise. The possible drawback may be the stringent requirement of hardware implementation. With the advent of low-cost DSP-based microcontrollers, this stringent requirement can readily be overcome.

The direct PWM method is to produce a bipolar ultrasonic switching voltage waveform with a controllable pulse width. The pulsewidths of both the positive half-cycle and the negative half-cycle are the same, and are symmetrical to $\pi / 2$ and $3 \pi / 2$, respectively. Fig. 4 shows the PWM waveform at a typical duty ratio, namely, $25 \%$. Notice that the controllable range of this duty ratio is from $0 \%$ to $50 \%$. Because of the limited speed range when solely controlled by the duty ratio, the frequency is adopted as another control variable. In general, 3-5 discrete frequency values are sufficient to enable the direct PWM method offering piecewise-linear characteristics throughout the whole operating range. Also, the use of discrete frequencies can avoid to bother the high nonlinearities of frequency control.

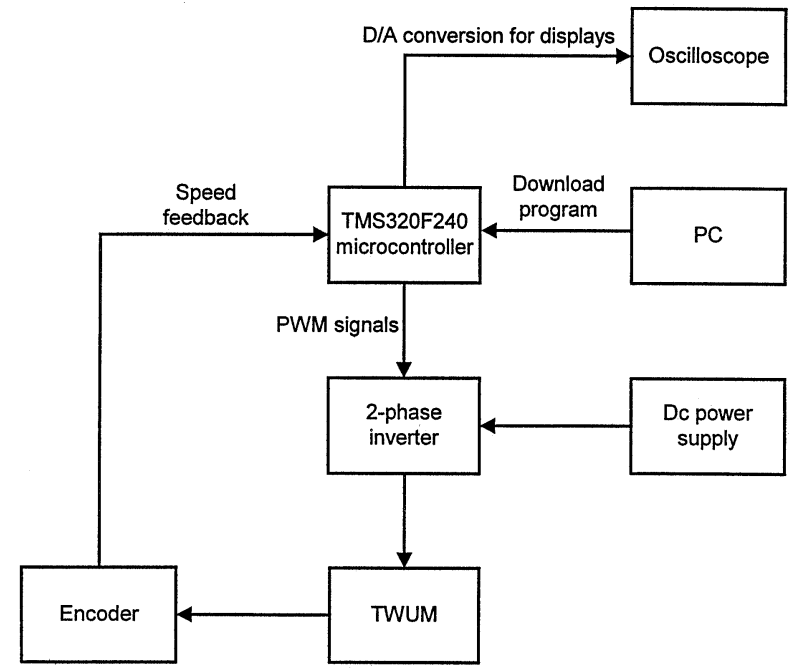

Fig. 5. System setup.

\section{SpeEd Tracking Control System}

Many published papers concerning motion control of the TWUM adopt a general servo control system for hardware implementation [12]-[17]. This system generally employs a microcomputer which commands a dual-channel function generator to provide proper switching signals for the two-phase inverter [4]. However, this system suffers from three key shortcomings which make it unrealistic for practical application. Firstly, the general purpose interface bus (GPIB), which links the microcomputer and the function generator, is not fast enough to provide good dynamic performance. Secondly, it is impractical to dedicate a standalone PC to control a TWUM. Thirdly, the required dual-channel function generator, having internal synchronization capability, is expensive and bulky.

Since the operating frequency of the TWUM is over $40 \mathrm{kHz}$, conventional microcontrollers are ill-suited for its motion control. Thus, a new DSP-based microcontroller TMS320F240 is selected as the core of hardware implementation. By using this TMS320F240, the sampling frequency for speed control can be greatly increased; hence, the dynamic performance can be significantly improved. Fig. 5 shows the setup of the proposed speed tracking control system. The control software is initially programmed by using the high-level language $\mathrm{C}$ in a PC. Then, the executable program is downloaded into the TMS320F240 which can run in the standalone mode (without the PC). This TMS320F240 can directly generate ultrasonic-frequency PWM signals with variable phase differences, variable frequencies, and variable duty ratios.

Fig. 6 shows the two-phase inverter circuit for the TWUM. Since the input terminals of the TWUM are inherently capacitive, two small inductors $\mathrm{L} 1$ and $\mathrm{L} 2$, both of $1 \mathrm{mH}$, are purposely added in series to its terminals so that resonance results. The corresponding resonance can offer an advantageous feature of soft switching, hence, reducing the switching loss and EMI. Power devices S1 and S3 connecting to the positive rail are p-channel IRF9640 MOSFETs, whereas S2 and S4 to the negative rail are $n$-channel IRF740 MOSFETs. Such an arrangement can minimize the required number of isolated dc supplies for MOSFET gate driving. 


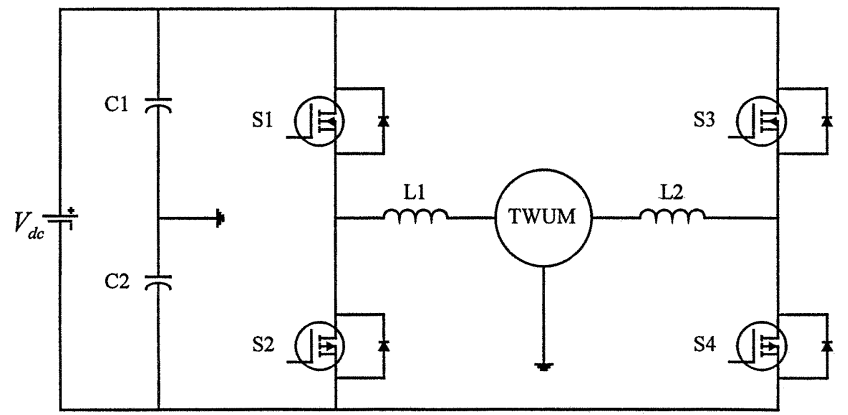

Fig. 6. Inverter circuit.

TABLE II

TWUM SPECIFICATIONS

\begin{tabular}{ll}
\hline Rated speed (rpm) & $90-100$ \\
Rated torque (Ncm) & 32 \\
Supply voltage range (V) & $100-130$ \\
Supply frequency range $(\mathrm{kHz})$ & $40-44$ \\
\hline
\end{tabular}

The overall control algorithm can be illustrated by the following steps.

Step 1) Sample instantaneous $N$; hence, calculate $\varepsilon$.

Step 2) Calculate $(\Delta f, \Delta d)$ using the proposed NFC algorithm.

Step 3) Update $f$ by $(f+\Delta f)$ and $d$ by $(d+\Delta d)$.

Step 4) Generate a PWM signal (based on the calculated $f$ and $d$ ) to drive $\mathrm{S} 1$ and $\mathrm{S} 4$.

Step 5) Generate another PWM signal (based on the calculated $f$ and $d$ but with $\phi=90^{\circ}$ ) to drive S2 and S3.

In order to assess the performance of the proposed dualmode NFC controller, both a dual-mode PI controller and a single-mode NFC controller are used for comparisons. The PI controller similarly adopts $f$ and $d$ as the dual-mode control variables, and also employs direct PWM. This serves to demonstrate the advantage of NFC over PI. Since there is no applicable mathematical model of the TWUM, the PI control parameters are chosen by trial and error. In this case, the PI constants are selected as $K_{p}=0.05$ and $K_{i}=4$. On the other hand, the single-mode NFC controller only adopts $f$ as the control variables. This serves to demonstrate the significance of using $f$ and $d$ as the dual control variables. The sampling time for both the PI controller and the NFC controllers is set to $10 \mathrm{~ms}$. The specifications of the TWUM are listed in Table II.

\section{EXPERIMENTAL RESULTS}

In order to assess the speed tracking capability of the proposed dual-mode NFC controller for the TWUM, two highly fluctuating speed commands (namely, square wave and sine wave) are used for experimentation. Figs. 7-9 show the speed tracking responses of the highly fluctuating square-wave command by using the dual-mode PI controller, the single-mode NFC controller, and the dual-mode NFC controller, respectively. It can be observed that the PI controller manages to reduce the steady-state error at the high speed level, but performs poorly at the low speed level. Owing to the high nonlinearities and limited resolution of frequency control, the

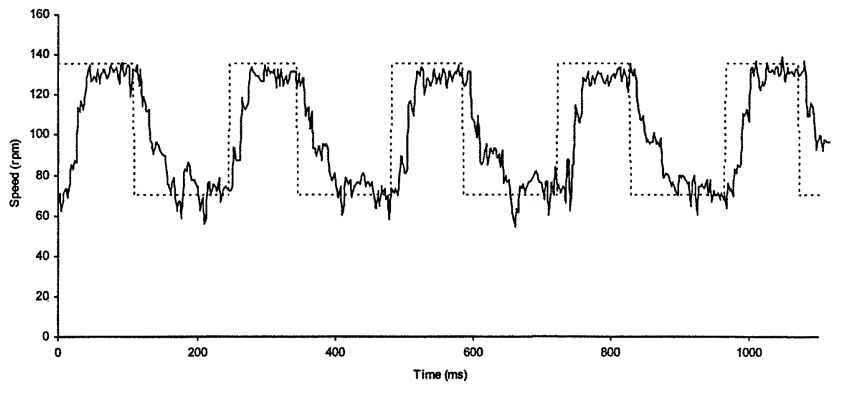

Fig. 7. Speed response by dual-mode PI control under square-wave command.

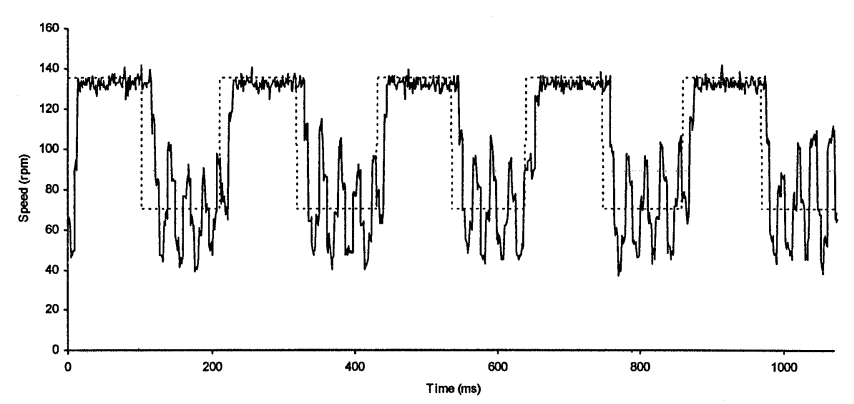

Fig. 8. Speed response by single-mode NFC under square-wave command.

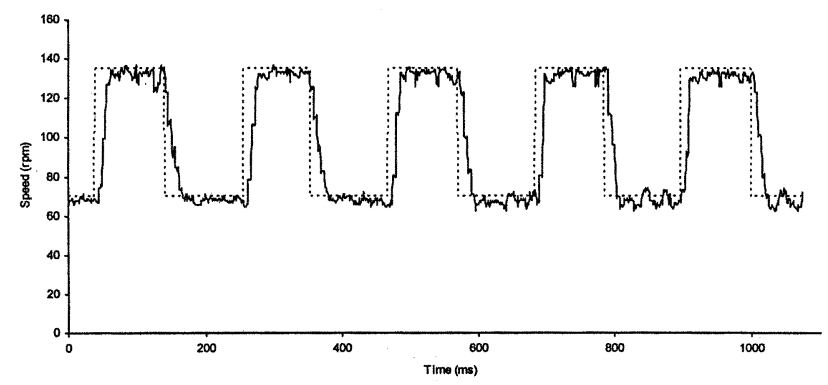

Fig. 9. Speed response by dual-mode NFC under square-wave command.

single-mode NFC controller also gives significant oscillations at the low speed. In contrast, the dual-mode NFC controller can exhibit a much better response. Particularly, there are much lower speed ripples at both the high speed and low speed levels.

To further demonstrate the adaptability of the proposed dual-mode NFC controller, the speed responses of the highly fluctuating sine-wave command by using the dual-mode PI controller, the single-mode NFC controller and the dual-mode NFC controller are shown in Figs. 10-12, respectively. As expected, the PI controller exhibits a very poor performance with serious speed ripples and significant phase delay. Although the single-mode NFC controller offers smaller speed ripples, it cannot solve the problem of significant phase delay. In contrast, the dual-mode NFC controller can offer a much better speed tracking response, which verifies that the proposed NFC approach can successfully adapt the change of command signals. 


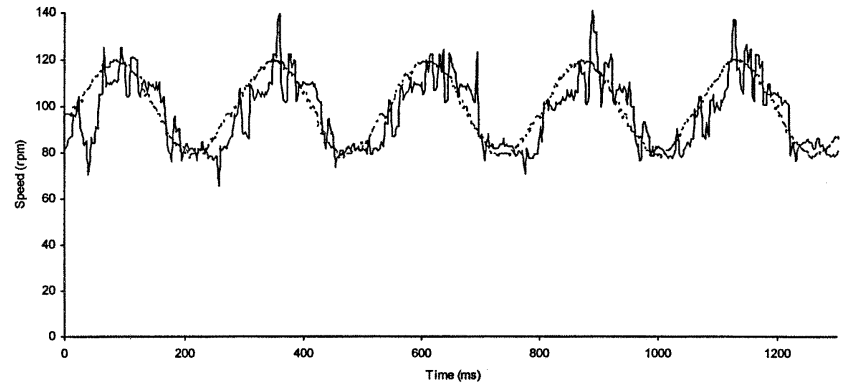

Fig. 10. Speed response by dual-mode PI control under sine-wave command.

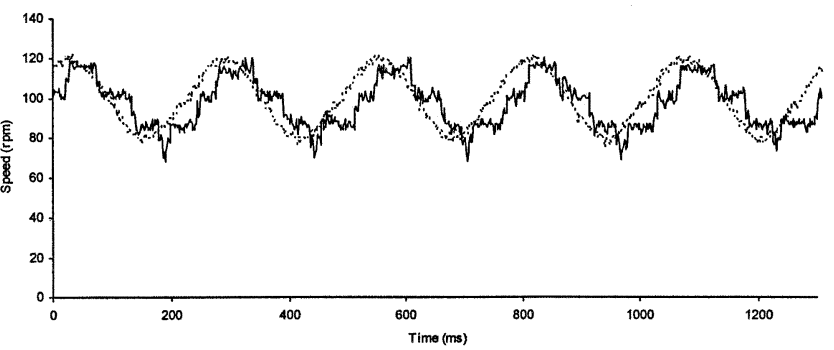

Fig. 11. Speed response by single-mode NFC under sine-wave command.

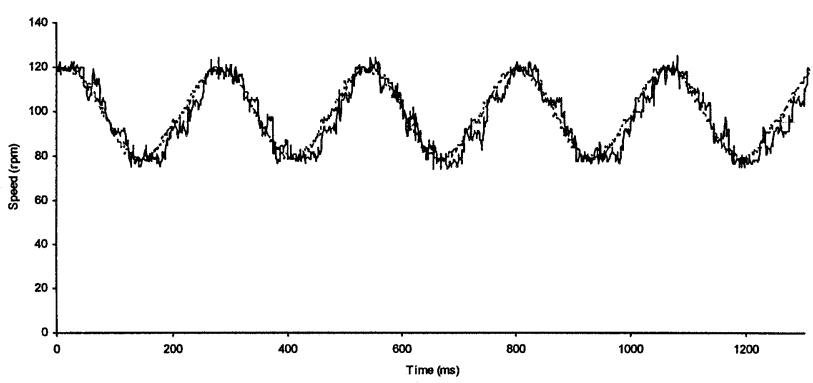

Fig. 12. Speed response by dual-mode NFC under sine-wave command.

Furthermore, the control signals of the proposed dual-mode NFC controller under the square-wave command are shown in Fig. 13. It verifies that both frequency control and duty ratio control work well during speed tracking.

\section{CONCLUSIONS}

In this paper, a new speed tracking control system using both NFC and direct PWM has been developed for the TWUM. It takes the advantages of using the NFC to handle the parameter variations and command adaptability, and using the direct PWM (based on dual-mode frequency and duty ratio control) to offer piecewise-linear characteristics. Experimental results demonstrate the superiority of the proposed dual-mode NFC controller over both the dual-mode PI controller and the single-mode NFC controller. Its adaptability to various command patterns is also verified.

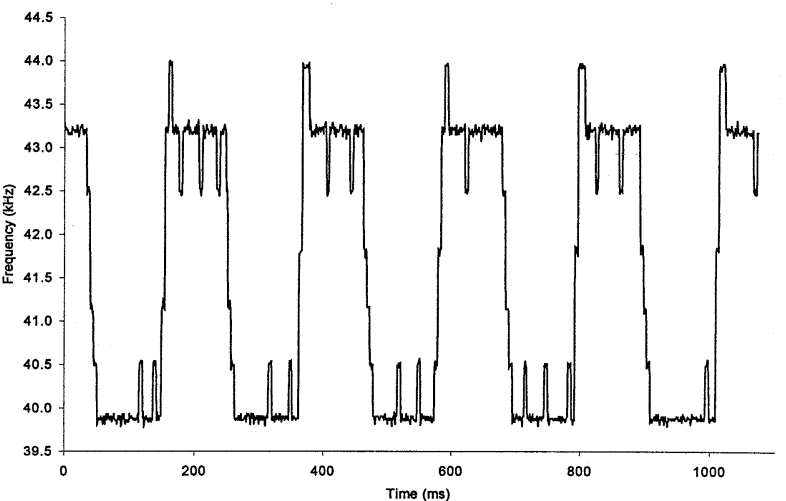

(a)

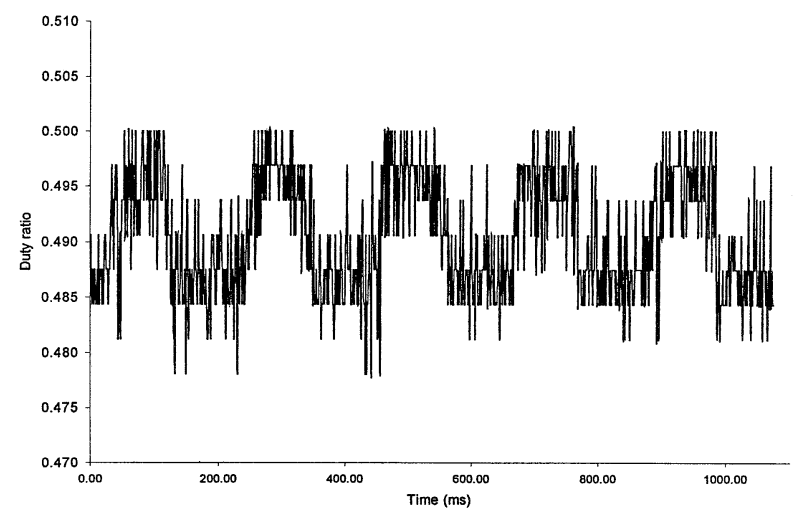

(b)

Fig. 13. Control signals of dual-mode NFC under square-wave command. (a) Frequency. (b) Duty ratio.

\section{REFERENCES}

[1] S. Ueda and Y. Tomikawa, Ultrasonic Motors Theory and Applications. Oxford, U.K.: Clarendon, 1993.

[2] T. Senjyu, H. Miyazato, and K. Uzeato, "Quick and precise position control of an ultrasonic motor with dual mode control," Int. J. Electron., vol. 80, no. 2, pp. 191-200, 1996.

[3] F. J. Lin, "Fuzzy adaptive model-following position control for ultrasonic motor," IEEE Trans. Power Electron., vol. 12, pp. 261-268, Mar. 1997.

[4] K. T. Chau and S.W. Chung, "Servo position control of ultrasonic motors using fuzzy neural network," Elect. Mach. Power Syst., vol. 29, no. 3, pp. 229-246, 2001.

[5] J. Mass and H. Grotstollen, "Cascaded control scheme for speed variable ultrasonic drives," in Conf. Rec. IEEE-IAS Annu. Meeting, 1996, pp. $440-447$.

[6] K. Nishibori, S. Okuma, K. Oshima, Y. Eryu, and Y. Ikegaya, "Position control of robot manipulators with ultrasonic motors using pulse width modulation," in Proc. IEEE IECON'96, 1996, pp. 1844-1849.

[7] K. Nishibori, S. Kondo, H. Obata, and S. Okuma, "PWM driving characteristics of robot hand with fingers using vibration-type ultrasonic motors," in Proc. IEEE IECON'97, 1997, pp. 1355-1360.

[8] S. W. Chung and K. T. Chau, "Servo speed control of traveling-wave ultrasonic motors using pulse width modulation," Elect. Power Comp. Syst., vol. 29, no. 8, pp. 707-722, 2001.

[9] N. W. Hagood and A. J. McFarland, "Modeling of a piezoelectric rotary ultrasonic motor," IEEE Trans. Ultrason., Ferroelect. Freq. Contr., vol. 42, pp. 210-224, Mar. 1995.

[10] P. Helin, V. Sadaune, C. Druon, and J. B. Tritsch, "A mechanical model for energy transfer in linear ultrasonic micromotors using Lamb and Rayleigh waves," IEEE/ASME Trans. Mechatron., vol. 3, pp. 3-8, Mar. 1998.

[11] P. A. Juang and W. Brenner, "The transfer function of a new disc-type ultrasonic motor," Sens. Actuators A, vol. 100, no. 2/3, pp. 272-280, 2002. 
[12] Y. Izuno, M. Hojo, and M. Nakaoka, "Software-based adjusting P-I gain controller for speed-tracking servo system using traveling-wave type ultrasonic motor," in Conf. Rec. IEEE-IAS Annu. Meeting, 1994, pp. $1777-1784$.

[13] S. I. Furuya, Y. Ohkura, and T. Maruhashi, "A novel inverter-drive ultrasonic motor-actuated positioning servo motion system using a fuzzy reasoning control scheme," J. Circuits, Syst. Comput., vol. 4, no. 4, pp. 395-414, 1994.

[14] T. Senjyu and K. Uzeato, "Adjustable speed control of ultrasonic motors by adaptive control," in Proc. IEEE PESC'94, vol. 2, 1994, pp. 1237-1242.

[15] T. Senjyu, H. Miyazato, S. Yokoda, and K. Uzeato, "Speed control of ultrasonic motors using neural network," IEEE Trans. Power Electron., vol. 13, pp. 381-387, May 1998.

[16] T. Senjyu, H. Miyazato, and K. Uzeato, "Speed control of ultrasonic motors using fuzzy neural network," in Proc. IEEE Int. Power Electronics Congr., 1996, pp. 29-34.

[17] F. J. Lin, R. J. Wai, and R. Y. Duan, "Fuzzy neural networks for identification and control of ultrasonic motor drive with LLCC resonant technique," IEEE Trans. Ind. Electron., vol. 46, pp. 999-1011, Oct. 1999.

[18] C. T. Lin and C. S. G. Lee, Neural Fuzzy System: A Neuro-Fuzzy Synergism to Intelligent Systems. Englewood Cliffs, NJ: Prentice-Hall, 1996.

[19] Y. Zhang, P. Sen, and G. E. Hearn, "An on-line trained adaptive neura controller,” IEEE Contr. Syst. Mag., vol. 15, pp. 67-75, Oct. 1995.

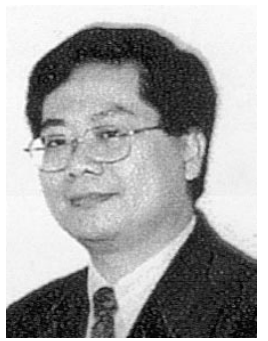

K. T. Chau (M'89) received the first-class honors B.Sc.(Eng.), M.Phil., and Ph.D. degrees in electrical and electronic engineering from The University of Hong Kong, Hong Kong, in 1988, 1991, and 1993 , respectively.

He is currently an Associate Professor at The University of Hong Kong. His teaching and research interests focus on three main areas: power converters, machines and drives, and electric vehicles. In these areas, he has authored more than 100 published refereed technical papers and several industrial reports He has also served as chair and organizing committee member for many international conferences. He is the coauthor of a monograph, Modern Electric Vehicle Technology (London, U.K.: Oxford Univ. Press, 2001).

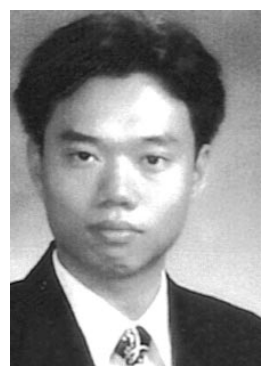

S. W. Chung was born in 1975 . He received the B.Eng. (Industrial Automation) degree in mechanical engineering in 1997 and the M.Phil. degree in electrical and electronic engineering in 2001 from The University of Hong Kong, Hong Kong, where he is currently working toward the Ph.D. degree in electrical and electronic engineering.

His research interests are servo motor drives and control theory application.

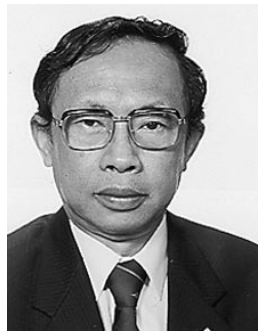

C. C. Chan (M'77-SM'77-F'92) received the B.Sc. degree from China University of Mining and Technology, Beijing, China, in 1953, the M.Sc. degree from Tsinghua University, Beijing, China, in 1957, and the Ph.D. degree from The University of Hong Kong, Hong Kong, in 1981.

He is currently Chair Professor in the Department of Electrical and Electronic Engineering, The University of Hong Kong. He has authored four books and more than 120 published technical papers and is the holder of seven patents.

Prof. Chan is a Fellow of the Royal Academy of Engineering, U.K., an Academician of the Chinese Academy of Engineering, and a Fellow of the Ukraine Academy of Engineering Science. He was awarded the Honorary D.Sc. degree from the University of Odessa in 1992. 